\title{
Microwave driven extraction of stabilized spin polarized atomic hydrogen
}

\author{
T. Roser, D.G. Crabb, W.A. Kaufman, R.S. Raymond, J.A. Stewart and B. Vuaridel \\ Randall Laboratory of Physics, The University of Michigan, Ann Arbor, Michigan 48109-1120, USA
}

G.R. Court

Department of Physics, Massachusetts Institute of Technology, Cambridge, MA 02139, USA, and

Department of Physics, Liverpool University, Liverpool L69 3BX,UK

Recerved 11 September 1990

\begin{abstract}
The storage of ultracold spin-polarized hydrogen atoms offers the possibility of producing a high intensity nuclear polarized atomic hydrogen jet. We stored electron spin polarized atomic hydrogen at $0.4 \mathrm{~K}$ in an open $5 \mathrm{~T}$ magnetic storage cell We also observed directly, for the first time, the extraction of hydrogen atoms from the storage cell by flipping their spins using a microwave driven transition. The results are being used to design a high intensity jet of nuclear polarized atomic hydrogen to be used as an internal target in the $400 \mathrm{GeV}$ to $3 \mathrm{TeV}$ UNK accelerator.
\end{abstract}

Spin effects in proton-proton collisions are now usually investigated with external polarized proton beams and solid cryogenic polarized proton targets. [1] A polarized proton gas jet target would allow such experiments to be performed with a circulating beam in a storage ring. Since a jet target contains only pure hydrogen, it eliminates the difficulty in discrimınating against the background from the bound nucleons in a conventional solid target. This greatly simplifies the study of exclusive reactions and is essential to the study of inclusive reactions. Present atomic beam production techniques [2] allow a maximum target thickness of approximately $10^{12}$ atoms $/ \mathrm{cm}^{2}$. For many high energy physics experiments this 1 too small by a factor of 100 to provide an adequate event rate. Methods of producing thicker polarized hydrogen gas targets have been recently reviewed [3].

Electron spin-polarized atomic hydrogen has been accumulated [4] in magnetic storage bottles with densittes in excess of $10^{18}$ atoms $/ \mathrm{cm}^{3}$. Kleppner and Niinikoski [5] proposed that a high density atomic hydrogen beam could be produced by using electron spin resonance (ESR) pumping to extract hydrogen atoms from a magnetic storage bottle. Such a microwave driven extraction process could provide an intense low velocity atomic beam with a narrow velocity distribution. With such a beam it should be possible to attain a target thickness of about $10^{14}$ atoms $/ \mathrm{cm}^{2}$ by using magnetic focusing. We report here the first direct observation of an electron spin-polarized atomic hydrogen beam formed by merowave extraction from a $0.4 \mathrm{~K}$ atomic hydrogen gas stored in a $5 \mathrm{~T}$ magnetic field [6]. The ESR transition was driven by $140 \mathrm{GHz}$ microwave radiation.

Electron spin-polarized atomic hydrogen can be stored at high densities over relatively long time periods since two hydrogen atoms with their spins aligned cannot recombine to form a molecule. Both storage and a high degree of electron spin polarization can be achieved. in a high magnetic field ( $\geq 5 \mathrm{~T})$ and at low temperatures $(\leq 0.5 \mathrm{~K})$ since the magnetic energy of a hydrogen atom, approximately $\mu_{\mathrm{e}} B$, is then considerably larger than the thermal energy $k T$. In an external magnetic field the four energy states of the hydrogen atom ground state are labeled a, b, c, and d in order of increasing energy. Atoms in the lower energy a and $b$ states are trapped in a high magnetic field region whereas the $c$ and $\mathrm{d}$ states are expelled [7].

Of the four possible transitions that convert $a$ or $b$ state atoms to $\mathrm{c}$ or $\mathrm{d}$ state atoms only the transitions a to $d$ and $b$ to $c$ are allowed one photon transitions. At a field of $5 \mathrm{~T}$ both transition frequencies are near 140 $\mathrm{GHz}$; their frequency dıfference is approximately equal to the hyperfine splitting of $1420 \mathrm{MHz}$. If either of these transitions is pumped, then $\mathrm{c}$ or $\mathrm{d}$ state atoms are generated in the trapping volume. These atoms diffuse into the field gradient regions at the ends of the volume; If the atoms do not recombine, they are then ejected 
forming a nuclear and electron polarized atomic beam with a velocity of about $240 \mathrm{~m} / \mathrm{s}$ and a narrow velocity spread of about $20 \mathrm{~m} / \mathrm{s}$. Spin exchange processes such as $d+b \leftrightarrow a+c$ with trapped $a$ and $b$ state atoms may reduce the nuclear polarization [8]. However, full electron spin polarization will still be maintained. Several authors $[8-10]$ have observed the decay of the $a$ and $b$ populations when shining resonant microwave radiation into closed storage cells where the ejection of the $c$ and d state was not possible.

Our apparatus, shown in fig. 1, was designed specifically to develop the technique of producing a high density atomic hydrogen beam by microwave driven extraction. The vertical section contained a continuous flow dilution refrigerator with a cooling power of 27 $\mathrm{mW}$ at $0.35 \mathrm{~K}$. The storage cell was a horizontal double-walled copper tube with an internal diameter of $48 \mathrm{~mm}$. The space between the two walls formed the mixing chamber of the dilution refrigerator. The storage cell was open at both ends and thus allowed the hydrogen feed and the microwave input hardware to be separated from the extraction region. The cell was mounted inside the bore of an $8 \mathrm{~T}$ superconducting solenoid, which has a magnetic field uniformity of $10^{-5}$ over a $10 \mathrm{~mm}$ diameter sphere.

Hydrogen atoms were produced in a rf dissociator.

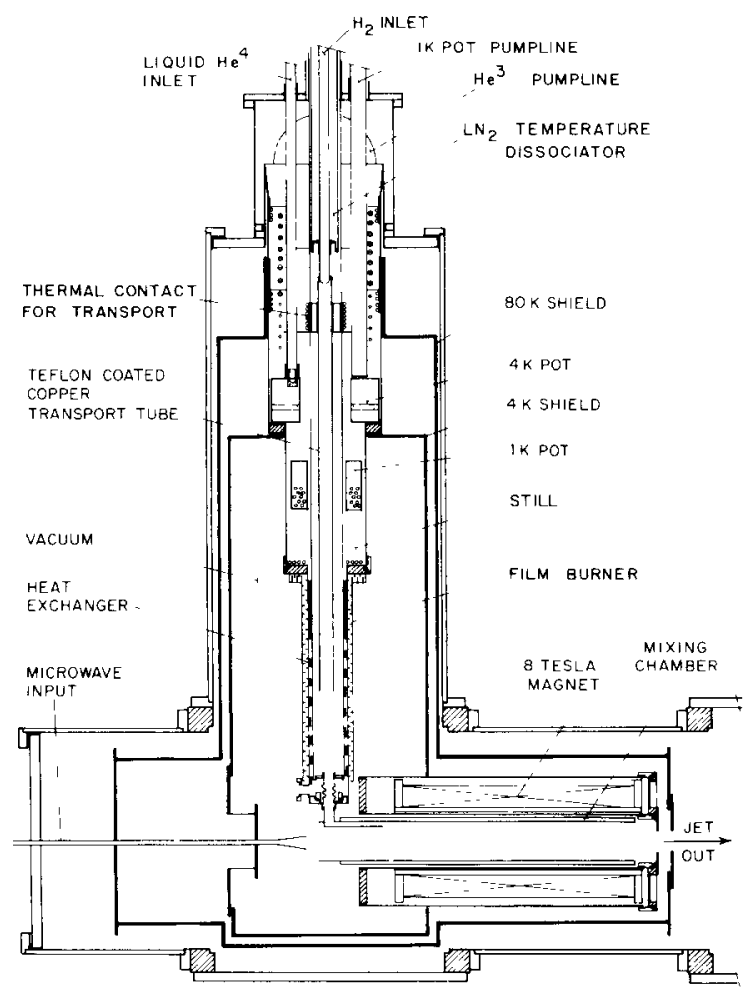

Fig. 1. Prototype jet apparatus.
They were cooled to a temperature of approximately 30 $\mathrm{K}$ and then guided to the low temperature region in a Teflon-coated copper tube. A fast temperature transition was then made to a tube which was cooled to a temperature of approximately $0.5 \mathrm{~K}$ by the final heat exchanger of the refrigerator. All cold surfaces were stably coated by a ${ }^{4} \mathrm{He}$ film with a $5 \mathrm{~nm}$ estimated thickness at a partial helium pressure of a few $10^{-4} \mathrm{~Pa}$. The helium film prevented rapid recombination of the atomic hydrogen [11].

The cold atoms were then guided into the fringe field of the solenoid where electron-spin selection takes place. Atoms in states $\mathrm{a}$ and $\mathrm{b}$ were accelerated towards the maximum field region at the center of the solenoid, while states $\mathrm{c}$ and $\mathrm{d}$ were repelled. After thermalization by gas and wall collisions states $a$ and $b$ were trapped axially by the magnetic field and radially by the cylındrical storage cell walls. Microwaves were injected from the left in fig. 1 and we detected atoms in states $\mathrm{c}$ or $\mathrm{d}$ that exited toward the right. At this extraction end the storage cell extended only to the point of maximum field gradient where the magnetic field is about half of the maximum field in the center. This represents a compromise between maximuzing the trapping efficiency and minimizing the number of wall collisions of the extracted atoms.

The microwave radiation, which was produced by a Varian Extended Interaction Oscillator, had a frequency in the range of 139 to $141 \mathrm{GHz}$ and a total power level of up to $60 \mathrm{~mW}$ at the horn. It was fed into the cell via a horn, which was cooled to $4.2 \mathrm{~K}$ and mounted $20 \mathrm{~mm}$ from one end of the storage cell. The microwave frequency stability was better than $5 \times 10^{-5}$.

We installed two types of detectors to observe the extracted atomic hydrogen beam. The thermal detector measured the thermal energy of $4.5 \mathrm{eV}$, which was released when two hydrogen atoms recombined on its surface. This detector was made of copper and operated at a temperature of $4.2 \mathrm{~K}$. At this temperature the specific heat of copper is very small and the sticking time of hydrogen atoms on the surface is long enough to ensure a high probability of recombination. For the intial extraction tests a solid $42 \mathrm{~mm}$ diameter disc was used. We later used a ring with a $44 \mathrm{~mm}$ outer diameter and an inner diameter of $22 \mathrm{~mm}$ together with the compression tube detector described below. A series of $10 \mathrm{~mm}$ deep circular baffles were soldered onto the backplane to increase the hydrogen capture probability. The thermal detectors were coupled to a $4.2 \mathrm{~K}$ heat sink via a thermal resistance which gave a $1 \mathrm{~K}$ temperature rise for an incident flow of $2.4 \times 10^{15}$ atoms/s assuming $100 \%$ recombination efficiency. The measured time constant of the thermal detectors was then approximately 4 s. The temperature rise was measured with a $\mathrm{Au}+0.07 \%$ Fe-Chromel thermocouple which gives an emf of 12.6 $\mu \mathrm{V} / \mathrm{K}$ at $4.2 \mathrm{~K}$. An electric heating wire was wound 


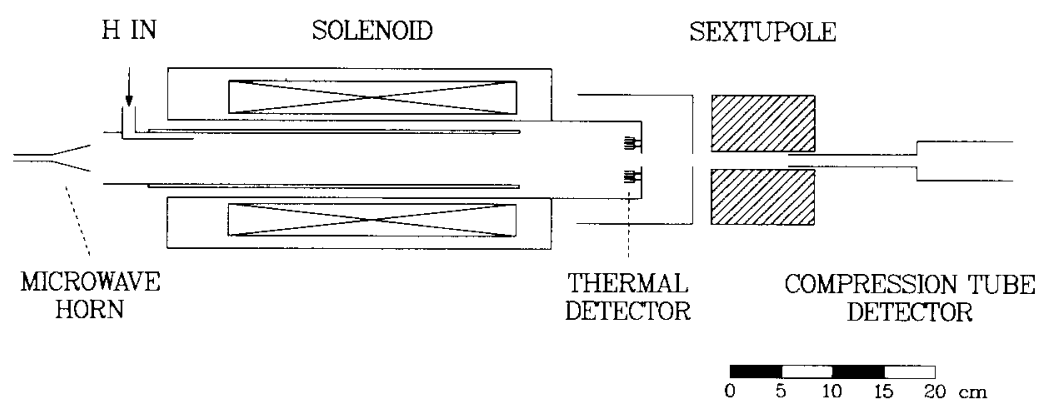

Fig. 2. Layout of the storage cell and extracted beam detectors.

around the rim of the detectors to allow periodic calibration.

The compression tube detector was located downstream of the storage cell as shown in fig. 2 ; it measured the increase of the partial pressure of molecular hydrogen in an enclosed volume whose only opening was a 1 $\mathrm{cm}$ diameter tube which was $19 \mathrm{~cm}$ long. Assuming that all the hydrogen atoms recombined, the molecular hydrogen pressure, as measured by a quadrupole mass spectrometer, was proportional to the flow of atomic hydrogen into the compression tube opening. The detector was calibrated by bleeding molecular hydrogen into the volume at a measured rate. The detector entrance was movable between $17 \mathrm{~cm}$ and $31 \mathrm{~cm}$ downstream from the end of the storage cell.

Since neither of these detectors gave information on the spin state of the extracted atoms, we installed a sextupole magnet spin filter which focused atoms in the $c$ and $d$ states and defocused the other states [12]. Hence, the focusing of extracted atoms by the sextupole was a clear signal for the presence of the $c$ or $d$ state atoms. The sextupole magnet was $10 \mathrm{~cm}$ long with 15 $\mathrm{mm}$ bore iron pole tips, and was located to overlap the movement range of the compression tube detector.

We first mounted the disc-shaped thermal detector with its face $2 \mathrm{~cm}$ downstream of the storage cell. Even with no resonant microwave radiation we observed a signal on the thermal detector due to hydrogen atoms leakıng out of the storage cell. With a magnetic field of $5 \mathrm{~T}$ at the center of the solenoid, the signal decayed with a time constant of approximately $21 \mathrm{~s}$ after the hydrogen feed was turned off. The decay time at zero field was shorter than the $4 \mathrm{~s}$ time constant of the detector. This demonstrated clearly that storage did occur.

The first extraction tests were made with continuous inputs of both microwave power and atomic hydrogen. The $140 \mathrm{GHz}$ microwave radiation itself produced a very stable signal in the thermal detector of about 0.4 $\mathrm{mW}$ when the microwave power level at the horn was 30 $\mathrm{mW}$. The magnetic field was then ramped through the ESR transitions near 5 T. Fig. 3 shows a typical signal from the thermal detector obtained by first raising and then lowering the magnetic field. The two peaks in each ramp correspond to the $\mathrm{a} \rightarrow \mathrm{d}$ and $\mathrm{b} \rightarrow \mathrm{c}$ transitions [7]. The separation of the peaks in fig. 3 was measured to be $510 \pm 10 \mathrm{G}$, agreeing well with the calculated separation of $507 \mathrm{G}$ or $1420 \mathrm{MHz}$ due to the hyperfine splitting. The sharp edges at the low field side of the peaks correspond to the rapid change of hydrogen extraction as the center of the trap suddenly passes into or out of the resonance condition. In the tail on the high field side, the resonance region slowly moved towards the edges of the trap. We compared the size of the peaks with electric pulses applied to the calibration heater of the thermal detector. We observed repeatedly peaks that corresponded to an instantaneous flow of $10^{16}$ atoms $/ \mathrm{s}$ [13].

We used the combination of the compression tube detector and the $15 \mathrm{~mm}$ bore sextupole magnet to determine the electron spin state population of the extracted hydrogen atoms. When both the microwave frequency and the magnetic field were kept fixed to drive the $\mathrm{a} \rightarrow \mathrm{d}$ transition, hydrogen atoms were extracted continuously. We simultaneously recorded the

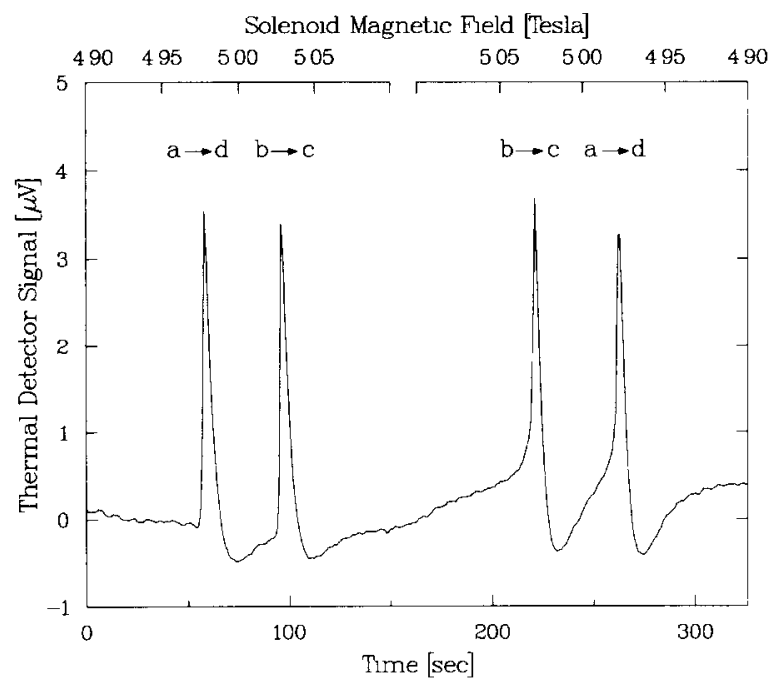

Fig. 3. Thermal detector response for a ramped magnetic field. 


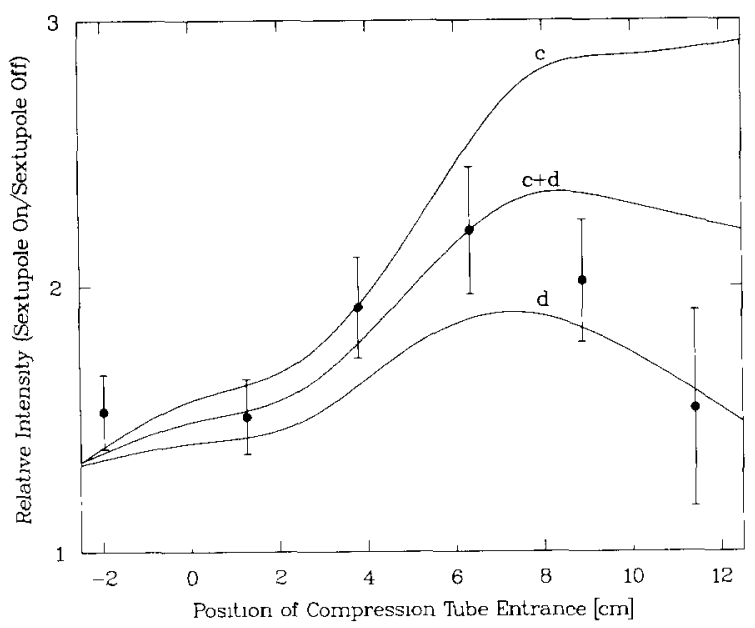

Fig. 4. The sextupole-on to sextupole-off ratio of the compression tube detector response is plotted against the detector position measured from the upstream face of the sextupole.

The errors show the variations of repeated measurements

signal from the ring-shaped thermal detector and used it to correct for changing hydrogen feed conditions. Fig. 4 shows the sextupole-on to sextupole-off ratio of the atomic beam flow measured along the beam axis with a sextupole pole tip field of $0.12 \mathrm{~T}$. The observed focusing effect due to the sextupole clearly demonstrates the presence of $c$ and $d$ state atoms. The three curves in fig. 4 are the result of a calculation [14], which tracked c, d and an equal mixture of $c$ and $d$ state atoms from the center of the storage cell to the detector. The data points are in good agreement with the tracking calculations demonstrating not only that the extracted hydrogen atoms are electron spin polarized, but also that they have the expected velocity of $240 \mathrm{~m} / \mathrm{s}$. The data may also suggest that the extracted atoms are still mostly in the $\mathrm{d}$ state and thus still have nuclear polarization. If true, this would suggest that spin exchange processes were not important.

Thus, we have directly observed for the first time an extracted beam of electron spin polarized atomic hydrogen obtained by driving an ESR transition with microwaves. The measured properties of the microwave extracted atomic beam are in good agreement with the expected formation of a slow, monoenergetic jet of spın polarized hydrogen atoms. Nuclear polarization can then easily be obtained by a standard rf transition unit as is used in a conventional atomic beam source. If the emerging atoms were magnetically focused into a $0.5 \mathrm{~cm}$ wide beam spot the measured flow of $10^{16}$ atoms $/ \mathrm{s}$ would result in a thickness of approximately $10^{12}$ atoms $/ \mathrm{cm}^{2}$. These first results, obtained with this prototype device, are already comparable with densities obtained with conventional atomic beam sources. We are using these prototype studies to help design a high density jet. We plan to use this high density ultracold spin polarized atomic hydrogen jet as an internal target in the $400 \mathrm{GeV}$ to $3 \mathrm{TeV}$ UNK accelerator at Protvino, USSR, as part of the NEPTUN-A [15] and NEPTUN [16] experıments which will study spin effects in proton-proton scattering.

\section{Acknowledgements}

We wish to thank Professors A.D. Krisch, D. Kleppner, and T.J. Greytak for their support and advice and Dr. T.O. Niinıkoski and P.R. Cameron for their help in the early stages of the project. We also thank Brookhaven National Laboratory for providing laboratory space and support. This work was supported by grants from the U.S. Department of Energy.

\section{References}

[1] R.C. Fernow and A.D. Krisch, Annu. Rev Nucl. Part. Sci. 31 (1981) 107.

[2] P.A. Schmelzbach, D. Sıngy, W. Grüebler and W.Z. Zhang, Nucl Instr. and Meth. A251 (1986) 407.

[3] W. Haeberl, Proc. 8th Int. Symp on High Energy Spin Physics, Minneapolıs, 1988, ed. K. Heller, AIP Conf. Proc. 187 (1988) 1487.

[4] H.F. Hess, D.A. Bell, G.P. Kochansk1, D. Kleppner and T. Greytak, Phys. Rev. Lett. 51 (1983) 483;

R. Sprik, J.T.M. Walraven and I.F. Silvera, Phys. Rev. Lett. 51 (1983) 479 and 51 (1983) 942.

[5] T.O. Nınıkoskı, Proc 4th Int. Symp. on High Energy Physics with Polarized Beams and Polarized Targets, Lausanne, 1980, (EXS 38, Burkhàuser, Basel and Stuttgart, 1981) p. 91;

D. Kleppner and T.J. Greytak, Proc. 5th Int. Conf. on High Energy Spin Physics, 1982, Brookhaven National Laboratory, ed. G.M. Bunce, AIP Conf. Proc. 95 (1983) p. 546.

[6] T. Roser, D.G. Crabb, W.A. Kaufman, R.S. Raymond, J.A. Stewart, B Vuandel and G.R. Court, Proc. Stanford 1989 Topical Conf. on Electronuclear Physics with Internal Targets, ed. R G. Arnold (World Scientıfic, Singapore, 1989) p. 183.

[7] T J. Greytak and D. Kleppner, in Course 11 of New Trends in Atomic Physics, Les Houches, Session XXXVIII, 1982, eds. G. Grynberg and R. Stora (Elsevier, 1984).

[8] T.O. Niınıkoski, S. Penttilä, J.M. Rıeubland and A. Rı]llart, Proc 4th Int. Workshop on Polarized Target Materıals and Techniques, 1984, University of Bonn, Bonn, FRG, 1984, ed. W. Meyer.

[9] A P.M. Matthey, J. van Zwol, J.T.M. Walraven and I.F. Silvera, Phys. Rev. B37 (1988) 4831.

[10] Ejected c state atoms have been observed in a closed cell by M.D. Hürlımann, W.N. Hardy, A.J. Berlınsky and R W. Cline, Phys Rev. A34 (1986) 1605 in their work on cryogenic hydrogen masers 
[11] I F. Silvera and J.T.M. Walraven, Phys. Rev. 44 (1980) 164.

[12] W. Haeberli, Annu. Rev. Nucl. Sci. 17 (1967) 373.

[13] We believe that we observed peaks corresponding to a flow of $4 \times 10^{16}$ atoms/s in an early phase of the exper1ment. Unfortunately, the records of this measurement have been misplaced.
[14] M. Ellilà, T.O. Niinikoskı and S. Penttılä, Nucl. Instr. and Meth. B14 (1986) 571

[15] A.D. Krisch, Proc. of Workshop Physics at UNK, IHEP, 1989, Protvino, USSR, p. 152.

[16] V.L. Solovianov, Proc. of Workshop on the experimental program at UNK, IHEP, 1987, Protvino, USSR, p 191. 